\title{
Influence of Home-Based Pulmonary Rehabilitation on Muscle Oxygenation in Elderly Patients with Chronic Obstructive Pulmonary Disease
}

JUNKO ODAGAWA RPT, MS ${ }^{1)}$, KIYOKAZU SEKIKAWA RPT, $\mathrm{PhD}^{2)}$, Kotaro KaWAGUCHI RPT, PhD ${ }^{3)}$, MAKOTO TAKAHASHI RPT, $\mathrm{PhD}^{2)}$, NAOKI MORITA MD, $\mathrm{PhD}^{4)}$, TSUTOMU INAMIZU MD, $\mathrm{PhD}^{2)}$

${ }^{1)}$ Shimoyama Memorial Clinic

${ }^{2)}$ Division of Physical Therapy and Occupational Therapy Sciences, Graduate School of Health Sciences, Hiroshima University: 1-2-3 Kasumi, Minami-ku, Hiroshima 734-8551, Japan. TEL: +81 82-257-5555

${ }^{3)}$ Institute of Rehabilitation, Hyogo University of Health Sciences

4)Kure Kyosai Hospital Tadanoumi Branch

\begin{abstract}
Purpose] The purpose of this study was to evaluate the benefit of 8-week home-based lower extremity strength training combined with whole body endurance training on peripheral muscle oxygenation in outpatients with chronic obstructive pulmonary disease (COPD). [Subjects] The 8 subjects were COPD patients ( 6 males, 2 females). [Methods] Using near infrared spectroscopy (NIRS), muscle oxygenation in the right vastus lateralis muscle at rest and during isometric contraction of $30 \%$ maximal voluntary contraction (MVC) were assessed before and after intervention. Also, MVC of the right quadriceps muscles, the muscle endurance time (TLIM), defined as the time from the onset of the isometric contraction of $30 \% \mathrm{MVC}$ until termination of the test, and the distance covered in the incremental shuttle walking test (ISWD) were measured. [Results] Although there were improvements, no significant changes were seen in MVC, TLIM, and ISWD between before and after the 8-week intervention. Also, no changes were seen in NIRS parameters at rest and during muscle endurance exercise between before and after intervention. [Conclusion] Our results identify no statistically significant improvements in MVC and walking ability which could be acknowledged as an effect of pulmonary rehabilitation. Further investigation is needed to clarify the effect of pulmonary rehabilitation on muscle oxygenation.
\end{abstract}

Key words: COPD, Near infrared spectroscopy, Muscle exercise therapy

(This article was submitted Apr. 9, 2009, and was accepted Jun. 5, 2009)

\section{INTRODUCTION}

There is growing body of evidence that skeletal muscle dysfunction such as decreases in muscle strength, endurance, and morphological changes are present in the skeletal muscle of COPD patients, and these dysfunctions show clinical relevance because they are related to an increased need for medical assistance ${ }^{1)}$. A statement was published by the American Thoracic Society in $1999^{2)}$ stating the significance of rehabilitation. The benefits of pulmonary rehabilitation include clinically relevant improvements of exercise capacity and healthrelated quality of life (HRQOL). Strength and endurance training of the ambulatory muscles is emphasized as there is accumulating evidence of its 
effectiveness for improving HRQOL. It has been reported that intervention of exercise therapy has a positive influence on muscle oxidative enzymes and recovery time of near infrared spectroscopy (NIRS) parameters ${ }^{3)}$. However, the influence of exercise therapy on skeletal muscle dysfunction as evaluated by NIRS has not yet been clarified.

The most available setting for pulmonary rehabilitation is outpatient programs which can be hospital-based or community-based. In Japan, most outpatient programs are hospital-based, as community-based programs are uncommon. Inpatient programs are also held for the sickest patients. Home-based programs where the practitioner visits the home are also rare in Japan.

The purpose of this study was to evaluate the benefit of home-based lower extremity strength training combined with whole body endurance training on peripheral muscle oxygenation in patients with COPD.

\section{SUBJECTS AND METHODS}

\section{Subjects}

The eight subjects were COPD patients (6 males, 2 females). Five patients had mild COPD and 3 patients had moderate COPD. Their average age, height and weight (mean \pm SD) were $78.3 \pm 3.8 \mathrm{yrs}$, $159.8 \pm 7.0 \mathrm{~cm}$ and $57.6 \pm 11.7 \mathrm{~kg}$, respectively.

This study was approved by the ethics committee of Hiroshima University Graduate School of Health Sciences. Informed consent was obtained from all subjects before their participation in this study.

\section{Methods}

Muscle strength was evaluated by measuring maximal voluntary contraction (MVC) of the right quadriceps muscles using a hand-held dynamometer (Power Track II, JTECH, USA). Subjects were seated upright with a hip flexion of 90 degrees and knee flexion of 30 degrees. They performed three maximal contractions and the highest value of these three contractions was considered the MVC. A steady maximal contraction, with a plateau phase of at least 2 seconds was necessary for MVC.

A near infrared spectrophotometer (Omega Monitor BOM-L1TRW, Omega Wave, Japan) was used to monitor tissue oxygen saturation. Three wavelengths $(780,810$, and $830 \mathrm{~nm})$ were monitored. Photo-detectors absorb the three wavelengths from a light source, and are analyzed using a modified versions of the Lambert-Beer ${ } \mathrm{aw}^{4)}$. By using these three wavelengths, it is possible to differentiate between oxygenated and deoxygenated hemoglobin $(\mathrm{OxyHb}$ and $\mathrm{DeoxyHb}$, respectively). The sum of $\mathrm{OxyHb}$ and DeoxyHb reflects the total amount of hemoglobin (TotalHb), which can be interpreted as blood volume in the underlying tissue. The depth of measurement can be specified, when using the BOM-L1TRW. Thus, the influence of adipose tissue, skin blood volume and flow can be excluded.

The two photo-detectors were placed at $20 \mathrm{~mm}$ and $40 \mathrm{~mm}$ from the light source. The photondepth penetration was therefore $10-20 \mathrm{~mm}$ below the surface of the skin. The NIRS unit was calibrated prior to each test. The NIRS probe was positioned on the right vastus lateralis muscle $10 \mathrm{~cm}$ from the superior region of the patella during measurements of quadriceps muscle endurance. The probe and detector were set in an optically dense rubber holder to ensure the stability of their positions relative to each other, and were secured to the thigh using adhesive tape to minimize motion artifacts and contamination of the signal by ambient light.

A weight that corresponded to $30 \% \mathrm{MVC}$ was placed on the subject's right ankle. The trial was initiated with a 2-minute baseline recording with the subject sitting with the right knee flexed at a resting position of 90 degrees. During the test, subjects were told to extend the right leg from the resting position to 30 degrees of knee flexion. An alignment bar was used for subject feedback. The subject received encouragement during the test, but was not informed of the elapsed time. An occlusion cuff (E20 Rapid Cuff Inflator, Hokanson, USA) was placed on the proximal end of the right thigh, and rapidly inflated during the muscle endurance test to a pressure of $30 \mathrm{mmHg}$ higher than the right leg systolic blood pressure, which was measured prior to the muscle endurance test, and was sharply deflated at termination of the test. The test was terminated at volitional fatigue, intolerable pain, intolerable numbness, or if the subject lost contact with the alignment bar. The time from the onset of the isometric contraction until termination of the test was recorded as the muscle endurance time (TLIM).

Resting tissue oxygen saturation $\left(\mathrm{StO}_{2}\right)$, measured by NIRS, was calculated as $\mathrm{OxyHb} /$ TotalHb $\times 100$. The ratio between pre-muscle 
Table 1. Changes of MVC, TLIM and NIRS during intervention

\begin{tabular}{lccc}
\hline & pre- PR & 4wks PR & 8wks PR \\
\hline MVC (kg) & $18.7 \pm 2.0$ & $18.7 \pm 3.1$ & $19.0 \pm 2.6$ \\
TLIM (s) & $267.0 \pm 80.0$ & $291.0 \pm 88.9$ & $358.2 \pm 76.3$ \\
$\Delta$ Oxy Hb (\%) & $-22.8 \pm 20.6$ & $-24.0 \pm 19.1$ & $-25.4 \pm 18.9$ \\
$\Delta$ Deoxy Hb $(\%)$ & $56.0 \pm 19.3$ & $68.7 \pm 26.9$ & $81.6 \pm 36.4$ \\
\hline
\end{tabular}

PR : pulmonary rehabilitation

endurance exercise and post-muscle endurance exercise was calculated for each NIRS artefact, and are shown as $\Delta \mathrm{OxyHb}, \Delta \mathrm{DeoxyHb}$ and $\Delta \mathrm{TotalHb}$, respectively.

The Incremental Shuttle Walking Test (ISWT) was performed to measure walking performance. The ISWT is a maximal, symptom-limited field exercise test which was developed by Singh ${ }^{5)}$. It is an externally paced test in which subjects are instructed to walk around a $10 \mathrm{~m}$ course at a speed indicated by beeps played from a CD player. There are 12 levels to this test. At level 1, subjects walk around the 10 meter course 3 times. Ten meters are added each minute as the level increases. The speed increases incrementally until the patient is unable to continue or maintain the required speed. The distance covered in the ISWT was recorded as shuttle walking distance (ISWD). The test was administered before intervention and after completion of the rehabilitation program.

Patients underwent an 8-week pulmonary rehabilitation program which included lower limb strength training and aerobic training. Training was carried out at least 3 days a week with at least one supervised session. Subjects recorded the unsupervised sessions in a home-training diary.

A Theraband ${ }^{\circledR}$ Exercise Band (Thera-Band, USA) was used for lower limb strength training. The exercise band was 1.3 meters in length and was cut from a standard commercial roll. The strength of the bands used were extra heavy (blue) and super heavy (silver) bands. The level of resistance was determined by using the DeLorme method ${ }^{6)}$ and the exercise bands were chosen according to each patient's strength. The patient was instructed to do 10 repetitions per set, and 3 sets of each exercise. Ten repetitions are equivalent to approximately $80 \%$ of 1 repetition maximum. The training included knee extension, hip flexion, and hip abduction exercises. The strength of the Theraband ${ }^{\circledR}$ was adjusted by changing the lever or by using a stronger exercise band to maintain $80 \%$ of maximum force.

For the aerobic training program, patients were instructed to walk for 20 minutes at a speed equal to $70-80 \%$ of the maximal speed attained on the ISWT. Seventy percent was prescribed if the patient was only able to complete the first half of a level, and $80 \%$ was prescribed if the patient was able to complete the latter half.

Data was collected preceding intervention of pulmonary rehabilitation (pre-PR), at 4 weeks (4wks-PR), and at 8 weeks ( 8 wks-PR). A repeatedmeasures analysis of variance was performed, and the Tukey-Kramer's post hoc test was used for pairwise comparisons. The paired t-test were used to determine the difference in ISWD between prePR and 8 wks-PR. Significance was accepted when $\mathrm{p}<0.05$. All statistical analyses were completed using statistical software (SPSS version 13.0J for Windows, SPSS Japan, Japan). Values are reported as mean \pm S.D..

\section{RESULTS}

Although there were improvements, no significant changes were seen in TLIM or MVC (Table 1).

The mean values for ISWD were $177.5 \pm 72.6 \mathrm{~m}$ at pre-PR and $241.3 \pm 82.9 \mathrm{~m}$ at $8 \mathrm{wks}-\mathrm{PR}$, with no significant change after intervention.

No changes were seen in resting $\mathrm{StO}_{2}$ of NIRS parameters during or after intervention of pulmonary rehabilitation $(50.2 \pm 8.0 \%, 52.6 \pm$ $6.6 \%, 50.4 \pm 8.3 \%$, respectively $\mathrm{p}=0.261)$. There were also no significant changes in NIRS parameters during muscle endurance exercise during or after intervention (Table 1). 


\section{DISCUSSION}

The purpose of this study was to evaluate the benefit of lower extremity strength training combined with whole body endurance training on peripheral muscle oxygenation using NIRS. However, no significant changes were found in muscle oxygenation or other clinical parameters. Whole-body exercise and local strength training are the cornerstones of exercise training in rehabilitation programs for COPD patients. It remains unclear whether whole-body endurance training, local muscle training, or a combination of both is preferable. As for exercise intensity, higher intensity training is emphasized as it is known that an overload is needed to achieve an adequate training stimulus ${ }^{7)}$. The prevailing thought is that patients with COPD have a ventilatory limitation that precludes the aerobic training levels necessary for beneficial physiological adaptations. However, studies have demonstrated that COPD patients are able to exercise at high intensities using intermittent training. Moreover, it is said that greater improvements in maximal and submaximal exercise responses can be obtained after high intensity training ${ }^{8)}$.

Previous studies on the effect of local muscle strength training in COPD have shown a positive effect on knee extensor strength ${ }^{9,10)}$. These studies prescribed exercise intensity at $60-85 \% \mathrm{MVC}$, the same as in this study. The major differences between the present study and previous ones are the program setting (home-based vs outpatient) and the equipment used. Home-based rehabilitation programs have been proven as effective as outpatient programs ${ }^{11)}$. The subjects in this study kept a home-training diary which was shown to the physiotherapist once a week on the day of the supervised session. Therefore, the training frequency of at least 3 times a week was administered properly. A Theraband ${ }^{\circledR}$ Exercise Band was used for lower extremity strength training as it has a number of advantages such as portability and affordability compared with free weights or machine weights. These elastic bands have been used in previous studies for orthopaedic rehabilitation ${ }^{12}$ ) and strength training for the elderly ${ }^{13-15)}$. Their major disadvantage is that it is difficult to quantify the load accurately. In this study, no increase was seen in MVC. This may have been due to insufficient load on the quadriceps muscle. However, no studies to our knowledge have reported the outcome measures of strength training or home-based rehabilitation although there have been reports of improvements in quality of life following strength training ${ }^{16)}$.

Endurance training is usually targeted at 60 to $90 \%$ of the predicted maximal heart rate or 50 to $80 \%$ of the maximal oxygen uptake ${ }^{2}$. In this study the ISWT, which is an objective, reliable and predictive test was used to assess maximal exercise performance. Patients were instructed to walk 20 minutes at $70 \%$ or $80 \%$ of the maximal speed achieved during the ISWT. They trained on a treadmill during supervised sessions in order to learn the prescribed speed for home training. Insignificant increases in distance of the ISWT were seen following rehabilitation.

NIRS has been shown to reflect changes before and after intervention of training. Neary et al. ${ }^{17)}$ reported significant increases in aerobic capacity and a greater mean muscle deoxygenation in already trained individuals. In their study, a short-term, highintensity cycling training program was administered for 3 weeks. Costes et al. ${ }^{18)}$ also reported a lower decrease of $\mathrm{StO}_{2}$ during mild to high intensity exercise. In their study, the untrained subjects trained 6 days a week for 4 weeks at $70-80 \%$ of the subject's maximal heart rate. Improvements were seen in muscle re-oxygenation kinetics assessed by NIRS accompanied with significant increases in exercise capacity following high intensity exercise training.

No changes were seen in NIRS parameters during rest or muscle endurance exercise in this study. In healthy people it has been shown that endurance training increases the vascularization of the muscle fibers and therefore, very likely local blood flow. The same may occur after training in COPD patients. The number of capillaries per unit surface area in the vastus lateralis muscles has been reported to be lower in COPD patients than age matched subjects. Capillary density appears to be dependent on the physical fitness of the subject and is known to be partly reversible with exercise training in previously untrained subjects. If the muscles had been subjected to a sufficient training load, changes in NIRS parameters may have been noted.

In conclusion, this study investigated the outcomes of home-based pulmonary rehabilitation and its influences on muscle oxygenation using 
NIRS. However, no significant changes were seen in muscle strength, endurance, or exercise capacity. Hence, no changes were seen in resting $\mathrm{StO}_{2}$ or $\Delta \mathrm{OxyHb}$ and $\Delta$ Deoxy during muscle endurance exercise. There were no statistically significant improvements in MVC or walking ability which are recognized measures of pulmonary rehabilitation. Further investigation is needed to clarify the effect of pulmonary rehabilitation on muscle oxygenation.

\section{REFERENCES}

1) Coggan AR, Spina RJ, King DS, et al.: Skeletal muscle adaptations to endurance training in 60- to 70-yr-old men and women. J Appl Physiol, 1992, 72: 17801786.

2) ATS/ERS: Pulmonary Rehabilitation---1999. Am J Respir Crit Care Med, 1999, 159: 1666-1682.

3) Puente-Maestu L, Tena T, Trascasa C, et al.: Training improves muscle oxidative capacity and oxygenation recovery kinetics in patients with chronic obstructive pulmonary disease. Eur J Appl Physiol, 2003, 88: 580587.

4) Simonson SG, Piantadosi CA: Near-infrared spectrosocopy: clinical application. Critical Care Clinics, 1996, 12: 1019-1029.

5) Singh SJ, Morgan MD, Scott S, et al.: Development of a shuttle walking test of disability in patients with chronic airways obstruction. Thorax, 1992, 47: 10191024.

6) Fish DE, Krabak BJ, Johnson-Greene D, et al.: Optimal resistance training: comparison of DeLorme with Oxford techniques. Am J Phys Med Rehabil, 2003, 82: 903-909.

7) ACSMPS: American College of Sports Medicine Position Stand. The recommended quantity and quality of exercise for developing and maintaining cardiorespiratory and muscular fitness, and flexibility in healthy adults. Med Sci Sports Exerc, 1998, 30: 975-991.

8) Casaburi R, Patessio A, Ioli F, et al.: Reductions in exercise lactic acidosis and ventilation as a result of exercise training in patients with obstructive lung disease. Am Rev Respir Dis, 1991, 143: 9-18.
9) Bernard S, Whittom F, Leblanc P, et al.: Aerobic and strength training in patients with chronic obstructive pulmonary disease. Am J Respir Crit Care Med, 1999, 159: 896-901.

10) Ortega F, Toral J, Cejudo P, et al.: Comparison of effects of strength and endurance training in patients with chronic obstructive pulmonary disease. Am J Respir Crit Care Med, 2002, 166: 669-674.

11) Strijbos JH, Postma DS, van Altena R, et al.: A comparison between an outpatient hospital-based pulmonary rehabilitation program and a home-care pulmonary rehabilitation program in patients with COPD. A follow-up of 18 months. Chest, 1996, 109: 366-372.

12) Treiber FA, Lott J, Duncan J, et al.: Effects of Theraband and lightweight dumbbell training on shoulder rotation torque and serve performance in college tennis players. Am J Sports Med, 1998, 26: 510-515.

13) Gill TM, Baker DI, Gottschalk $M$, et al.: A prehabilitation program for physically frail community-living older persons. Arch Phys Med Rehabil, 2003, 84: 394-404.

14) Capodaglio P, Ferri A, Scaglloni G: Effects of a partially supervised training program in subjects over 75 years of age. Aging Clin Exp Res, 2005, 17: 174180.

15) Topp R, Mikesky A, Dayhoff NE, et al.: Effect of resistance training on strength, postural control, and gait velocity among older adults. Clin Nurs Res, 1996, 5: 407-427.

16) Boxall AM, Barclay L, Sayers A, et al.: Managing chronic obstructive pulmonary disease in the community. A randomized controlled trial of homebased pulmonary rehabilitation for elderly housebound patients. J Cardiopulm Rehabil, 2005, 25: 378-385.

17) Neary JP, McKenzie DC, Bhambhani YN: Effects of short-term endurance training on muscle deoxygenation trends using NIRS. Med Sci Sports Exerc, 2002, 34: 1725-1732.

18) Costes F, Prieur F, Feasson L, et al.: Influence of training on NIRS muscle oxygen saturation during submaximal exercise. Med Sci Sports Exerc, 2001, 33: 1484-1489 